\title{
Defensinas humanas y su función en cavidad oral
}

\author{
Marsol Ivani Ortiz-Casillas, ${ }^{*}$ Carlos Rogelio Alvizo-Rodríguez, ${ }^{\ddagger}$ \\ Yveth Marlene Ortíz-García,§ Martha Graciela Fuentes-Lerma,§,\| \\ Melva Gutiérrez-Angulo," Rocío Patricia Mariaud-Schmidt§
}

\begin{abstract}
RESUMEN
La cavidad oral proporciona un entorno cálido y húmedo que hace a la flora bucal normal extremadamente compleja y propicia para la coIonización por hongos, virus y bacterias, requiriendo múltiples tipos de defensas para prevenir infecciones. Los péptidos antimicrobianos son importantes contribuyentes para mantener el equilibrio entre la salud y la enfermedad, éstos realizan varias funciones esenciales para la defensa contra los microorganismos, lo que modifica la respuesta inflamatoria local y activa mecanismos de reacción inmunitaria adaptativa, participando de esta manera en la modulación de la respuesta inmunológica, que otorga especificidad funcional y los clasifica en diferentes familias, una de ellas las defensinas. En el presente reporte se hace una revisión de la función y expresión de las defensinas y su relación con la cavidad oral.
\end{abstract}

Palabras clave: Inmunidad innata, péptidos antimicrobianos, defensinas, cavidad oral.

\section{INTRODUCCIÓN}

La inmunidad innata es la primera línea de defensa contra las infecciones, ${ }^{1}$ la cual se caracteriza por ser corta, inmediata, inespecífica y por no tener memoria; ${ }^{2}$ dentro de sus componentes se encuentran barreras físicas como la piel y mucosas de las vías aéreas y digestivas que son el contacto inicial de los microorganismos patógenos, razón por la cual las

* Postgrado de Odontopediatría, Departamento de Clínicas Odontológicas Integrales, Centro Universitario de Ciencias de la Salud, Universidad de Guadalajara.

‡ Doctorado en Genética Humana, Centro Universitario de Ciencias de la Salud, Universidad de Guadalajara.

$\S$ Instituto de Investigación en Odontología, Departamento de Clínicas Odontológicas Integrales, Centro Universitario de Ciencias de la Salud, Universidad de Guadalajara.

\| Departamento de Ciencias de la Salud. Centro Universitario de los Altos, Universidad de Guadalajara.

Recibido: 24 de octubre de 2019. Aceptado: 04 de diciembre de 2019.

Este artículo puede ser consultado en versión completa en www.medigraphic.com/periodontologia

\begin{abstract}
The oral cavity provides a warm and wet environment that makes normal oral flora extremely complex and conducive to colonization by fungi, viruses and bacteria, requiring multiple types of defenses to prevent infections. Antimicrobial peptides are important contributors to maintain the balance between health and disease, these perform several essential functions for the defense against microorganisms, which modifies the local inflammatory response and activates adaptive immunologic reaction mechanisms participating in the modulation of the immune response, which gives functional specificity and classifies them in different families being one of them the defensins. In this report a review of the functions and expression of the defensins and their relationship with the oral cavity.
\end{abstract}

Keywords: Innate immune, antimicrobial peptides, defensins, oral cavity.

células epiteliales producen péptidos antimicrobianos (PAM's). ${ }^{3,4}$ Éstos realizan varias funciones esenciales para la defensa contra los microorganismos; y por su acción citotóxica directa sobre bacterias, hongos, parásitos y virus, modifican la respuesta inflamatoria local y activan mecanismos de acción inmunitaria adaptativa. ${ }^{5}$

\section{PÉPTIDOS ANTIMICROBIANOS}

Los PAM's son importantes contribuyentes para mantener el equilibrio entre la salud y la enfermedad y participan en procesos como la modulación de la respuesta inmune, angiogénesis y cicatrización. ${ }^{6,7}$ Éstos han sido identificados en vertebrados, invertebrados, plantas y bacterias; ${ }^{8}$ poseen propiedades estructurales dinámicas debido a la variabilidad de los residuos de aminoácidos, los cuales pueden ser cortos (20 a 50 residuos) o poseer una estructura grande (hasta 200 aminoácidos).9,10 También se caracterizan por ser anfipáticos con ambos dominios: hidrófobos e hidrófilos, lo que facilita la unión a los componentes lipídicos y los fosfolípidos, respectivamente. ${ }^{11}$ 
Los PAM's, la mayoría de ellos catiónicos, atacan las membranas de las células bacterianas y provocan la desintegración de la bicapa lipídica ${ }^{12}$ a través de la interacción de la membrana celular cargada negativamente; ${ }^{13,14}$ además se han identificado algunos PAM's a bajas concentraciones cuyo mecanismo de acción se lleva a cabo sin cambiar la integridad de la membrana ya que inhabilitan vías importantes de la célula como son la replicación del ácido desoxirribonucleico y la síntesis de proteínas. ${ }^{13,15}$

Los PAM's se clasifican en catelicidinas, histatinas y defensinas; ${ }^{16}$ en el presente artículo haremos una revisión bibliográfica de las últimas.

\section{Defensinas}

El nombre «defensin» fue otorgado por el profesor de medicina, Roberto I Lehrer en 1985; se deriva del latín defendo, que significa «repeler», en referencia a sus propiedades frente a microorganismos patógenos. ${ }^{17}$

Las defensinas son péptidos catiónicos no glicosilados, siendo la arginina el residuo catiónico primario. Poseen un peso molecular aproximado de 3.5 a $6 \mathrm{kDa} ;{ }^{18}$ contienen seis residuos de cisteínas que forman tres puentes disulfuro responsables de la estructura beta plegada, y son abundantes en células y tejidos que participan en la defensa del huésped contra infecciones microbianas. ${ }^{19}$

Las defensinas están clasificadas en las subfamilias $\alpha, \beta$ y $\theta$ con base en la posición de los puentes disulfuros presentes, ${ }^{20}$ pero únicamente las $\alpha \mathrm{y}$ $\beta$ defensinas están presentes en humanos, estos subtipos difieren entre sí por la longitud de los residuos de aminoácidos, la posición de los enlaces disulfuro y su expresión celular. ${ }^{21}$ En la Tabla 1 se mencionan todos los genes que codifican para las defensinas descritas, así como su localización cromosómica. ${ }^{22}$

Las $\alpha$-defensinas contienen de 30-50 aminoácidos, ${ }^{23}$ principalmente arginina y el puente disulfuro se forma entre el primero y el sexto residuo de cisteína $;^{24}$ son producidas por promielocitos, células precursoras de los neutrófilos, razón por la cual reciben el nombre de péptidos de neutrófilos humanos (HNP) por sus siglas en inglés. ${ }^{25,26}$

Existen seis alfa defensinas agrupadas en mieloides (HNP 1-4) y entéricas (HNP 5 y HNP 6). ${ }^{27}$ Éstas son producidas como pre-pro-péptidos de 110 residuos
Tabla 1: Nombre y localización cromosómica de los genes y pseudogenes de las defensinas. ${ }^{22}$

\begin{tabular}{|c|c|}
\hline Genes de $\alpha$-defensinas & Localización \\
\hline DEFA1 & $8 p 23.1$ \\
\hline DEFA3 & 8p23.1 \\
\hline DEFA4 & $8 p 23.1$ \\
\hline DEFA5 & $8 p 23.1$ \\
\hline DEFA6 & 8p23.1 \\
\hline Genes de $\beta$-defensinas & Localización \\
\hline DEFB131A & $4 p 16.1$ \\
\hline DEFB110 & $6 p 12.3$ \\
\hline DEFB112 & $6 p 12.3$ \\
\hline DEFB113 & $6 p 12.3$ \\
\hline DEFB114 & $6 p 12.3$ \\
\hline DEFB1 & 8p23.1 \\
\hline DEFB4A & 8p23.1 \\
\hline DEFB103A & $8 p 23.1$ \\
\hline DEFB103B & 8p23.1 \\
\hline DEFB104A & $8 p 23.1$ \\
\hline DEFB104B & 8p23.1 \\
\hline DEFB105A & 8p23.1 \\
\hline DEFB105B & $8 p 23.1$ \\
\hline DEFB106A & 8p23.1 \\
\hline DEFB106B & $8 p 23.1$ \\
\hline DEFB107A & 8p23.1 \\
\hline DEFB108A & $8 p 23.1$ \\
\hline DEFB109A & $8 p 23.1$ \\
\hline DEFB130A & $8 p 23.1$ \\
\hline DEFB115 & $20 q 11.21$ \\
\hline DEFB116 & $20 q 11.21$ \\
\hline DEFB117 & $20 q 11.21$ \\
\hline DEFB118 & $20 q 11.21$ \\
\hline DEFB119 & $20 q 11.21$ \\
\hline DEFB122 & $20 q 11.21$ \\
\hline DEFB124 & $20 q 11.21$ \\
\hline DEFB125 & $20 \mathrm{p} 13$ \\
\hline DEFB126 & $20 p 13$ \\
\hline DEFB127 & $20 \mathrm{p} 13$ \\
\hline DEFB128 & $20 p 13$ \\
\hline DEFB129 & $20 p 13$ \\
\hline DEFB132 & $20 \mathrm{p} 13$ \\
\hline eudogenes de $\beta$-defensinas & Localización \\
\hline DEFB109A & 8p23.1 \\
\hline DEFB117 & $20 q 11.21$ \\
\hline DEFB122 & $20 q 11.21$ \\
\hline
\end{tabular}


de aminoácidos. ${ }^{28}$ La expresión de HNP 1-4 es mayormente detectable en neutrófilos, pero también se expresan en monocitos/macrófagos, natural killer, algunas células T, células B y células dendríticas inmaduras. Las HNP 5 y 6 son expresadas por las células intestinales de Paneth y por las células epiteliales de los tractos genitourinarios masculinos y femeninos. ${ }^{29}$ Las alfa-defensinas tienen una expresión débil pero, en respuesta a una infección, los niveles de estos péptidos se incrementan hasta 10 veces. ${ }^{30}$

Por su parte las $\beta$-defensinas (hBD) tienen de 38 a 42 residuos de aminoácidos y tres enlaces disulfuro entre los seis residuos de cisteína de los péptidos maduros, ${ }^{31}$ la primera $\beta$-defensina se aisló a partir de células traqueales de bovinos en 1993 a la que llamaron péptido antimicrobiano traqueal, ${ }^{32}$ y en humanos la defensina humana $\beta-1$, fue identificada en 1995 a partir de hemofiltrados de pacientes con enfermedad renal, ${ }^{7}$ actualmente se han identificado seis miembros de esta familia, hBD-1 a hBD-6, respectivamente.

Las $\beta$-defensinas son interesantes como posibles agentes inmunomoduladores, porque algunas de ellas eliminan el patógeno sin elevar la inflamación. ${ }^{33} \mathrm{La}$ expresión de las $\beta$-defensinas se observa en los diferentes epitelios del cuerpo humano que contribuyen a la protección de las superficies de mucosas. ${ }^{34}$

La hBD-3 ha demostrado ser altamente activa contra microorganismos Gram-positivos y se expresa en placenta, músculo del corazón, músculo esquelético, timo fetal, esófago y tráquea. ${ }^{35,36}$

El mecanismo de acción de las $\alpha$ y $\beta$-defensinas, consiste en la interacción de cargas positivas de éstos y la carga negativa de las membranas de las bacterias y virus; una vez que interactúan entre sí, las defensinas se acumulan en la membrana de los microorganismos y causan despolarización, lo que induce su muerte. Las membranas celulares humanas son de carga neutral, lo que garantiza el contacto selectivo de las defensinas con patógenos del huésped. Anexo a esto, en las $\alpha$-defensinas existe un mecanismo de acción antiviral adicional, al interactuar con las células humanas, el cual puede reducir la replicación y transcripción del virus. ${ }^{37}$

\section{EXPRESIÓN Y FUNCIÓN DE LAS $\boldsymbol{\beta}$-DEFENSINAS EN DIVERSOS TEJIDOS DEL ORGANISMO}

Coretti y colaboradores demostraron que la expresión defectuosa de los PAM's particularmente de las defensinas, se encuentra relacionada con la enfermedad de Crohn, donde se observa una reducción de $\alpha$-defensinas en el íleon, y un incremento de las $\beta$-defensinas cuando esta enfermedad se encuentra en el colon. ${ }^{38}$

Otros sitios de expresión de las defensinas incluyen células epiteliales del testículo, intestino delgado, córnea, región mamaria, astrocitos y tejido gingival, entre otras. ${ }^{7}$

El péptido antimicrobiano más importante en la defensa epitelial ante infecciones es la $\beta$ defensina $1 .{ }^{39} D E F B 1$, es el único gen de las $\beta$-defensinas con expresión constitutiva en queratinocitos de la piel, células epiteliales del tracto respiratorio y urinario, además es capaz de regularse por medio de microorganismos o estímulos inflamatorios. ${ }^{40}$ Las más altas concentraciones de la $\beta$ defensina humana 1 se han encontrado en el riñón y en tracto reproductor femenino, especialmente en mujeres embarazadas. ${ }^{7}$ Desempeña un papel fundamental en la biología humana al proteger a los recién nacidos de enfermedades infecciosas como la diarrea, y a las madres de la inflamación de las glándulas mamarias durante la lactancia. ${ }^{41}$

Asimismo, las $\beta$-defensinas actúan en los queratinocitos como factor de protección contra la apoptosis en los epitelios, ${ }^{42}$ producen lisis de membrana, inactivan retrovirus (VIH) y Mycobacterium tuberculosis: $;^{7,43,44}$ también actúan como supresores tumorales en células cancerosas, induciendo la apoptosis mediada por caspasas, ${ }^{45,46}$ y participan como inmunomoduladores de células $\beta$ y como receptor de quimiocinas. ${ }^{40}$

\section{PARTICIPACIÓN DE LAS $\beta$-DEFENSINAS EN CAVIDAD ORAL}

Uno de los principales sitios de colonización para los microorganismos en la cavidad oral son los tejidos duros dentales y tejidos gingivales, estos sitios pueden servir como reservorio principal de enfermedades. ${ }^{47}$

La saliva es parte esencial de los mecanismos de defensa contra los cambios microbianos continuos en la cavidad oral. Sin embargo, esta actúa como fluido diagnóstico para detectar cambios inflamatorios inducidos por infección en el periodonto. ${ }^{48}$ Las células epiteliales orales expresan altos niveles de $\beta$-defensinas humanas 1-3 en el epitelio gingival, bucal, lengua, glándulas salivales, pulpa dental ${ }^{49,50}$ 


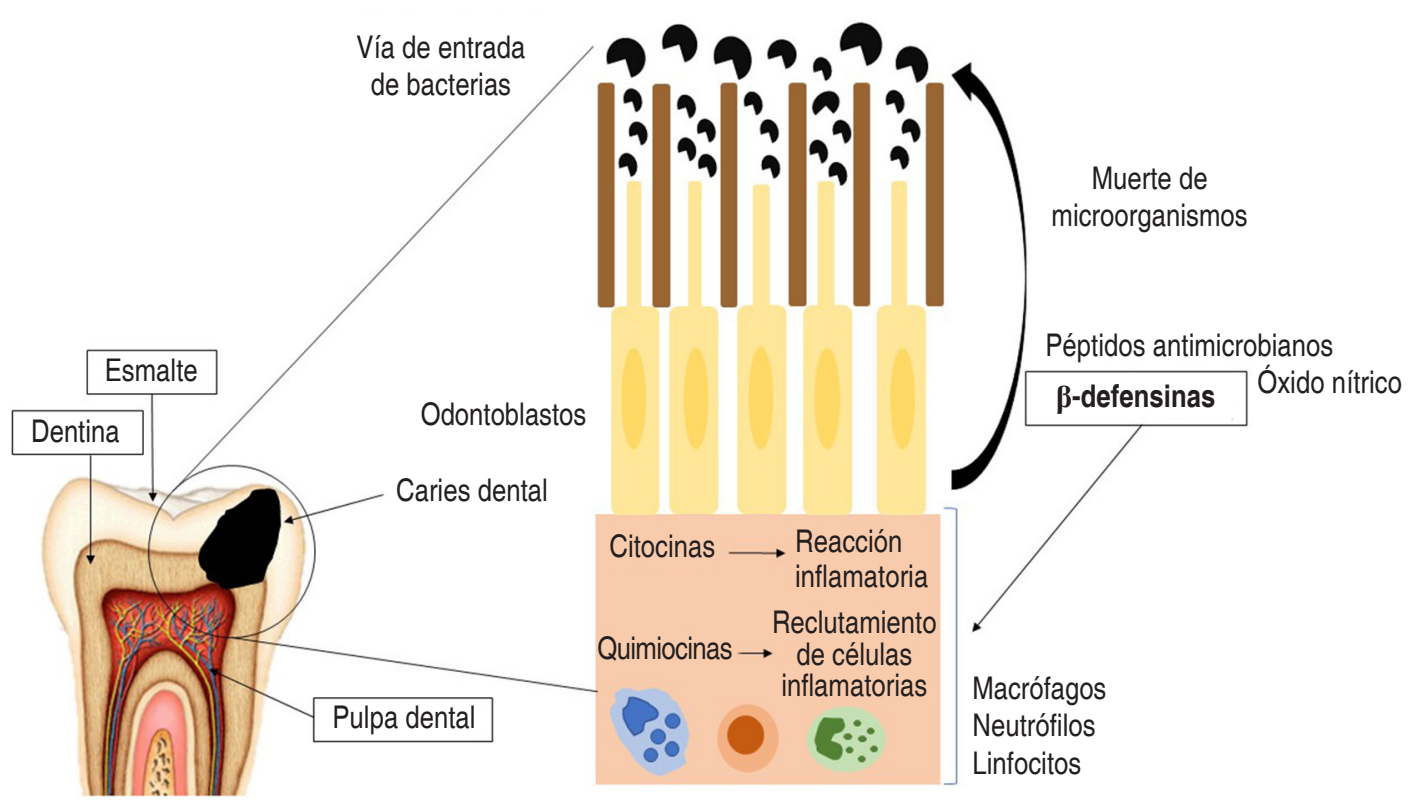

Figura 1: Función de las $\beta$-defensinas humanas en respuesta a los patógenos relacionados con caries dental.

y en carcinomas orales; ${ }^{51,52}$ las defensinas que se encuentran en ella juegan un papel importante contra una amplia gama de hongos y bacterias, son antioxidantes y mantienen la hemostasis de la boca. ${ }^{25}$

La $\beta$-defensina 1 es constitutivamente producida en glándulas parótidas, mucosa bucal, lengua, mucosa gingival, ${ }^{50,53}$ así como en células epiteliales ductales de glándulas salivales menores y la $\beta$-defensina 2 se expresa en queratinocitos de la mucosa gingival y glándulas salivales, ${ }^{54}$ ambas defensinas se encuentran presentes como antimicóticos durante la colonización por Candida albicans en el epitelio bucal; ${ }^{25}$ asimismo, Mckay y su grupo detectaron cantidades elevadas de $\alpha$-defensinas en el líquido crevicular gingival que se encuentra entre la superficie del diente y el epitelio gingival, las cuales cambian con la progresión y remisión de la inflamación periodontal. ${ }^{55}$

A su vez, Navarra y su equipo describen que la susceptibilidad a la caries dental entre la población general se atribuye a factores genéticos e inmunológicos, ${ }^{56}$ especialmente con respecto a varios componentes del sistema inmunitario innato que juegan un importante papel en la patogénesis de la enfermedad. ${ }^{57}$ Se ha demostrado que las $\beta$-defensinas poseen actividad antibacteriana contra los microorganismos relacionados con la caries ${ }^{58,59}$ y también desempeñan papeles importantes en la defensa pulpar del diente. ${ }^{60}$

El primer mecanismo de defensa contra la infección por microorganismos está relacionado con los odontoblastos, éstos forman una barrera natural entre los tejidos mineralizados y tejidos blandos del órgano dentario como la pulpa dental, ubicados en la parte más externa de esta última. Por lo tanto, los odontoblastos poseen un sistema inmune innato especializado para combatir los patógenos orales que invaden la dentina. ${ }^{61,62}$

Una vez que los receptores expresados en la membrana de los odontoblastos detectan a los microorganismos, se activa una vía de señalización que conduce a la producción y liberación de péptidos antimicrobianos (como las defensinas) y de óxido nítrico, éstos eliminan bacterias, virus, parásitos y hongos $;{ }^{61,62}$ la liberación de $\beta$ defensinas induce la producción de quimiocinas para atraer células inflamatorias al sitio de infección, como se observa en la Figura 1.

Además, se han demostrado funciones específicas de las $\beta$-defensinas en la regulación del crecimiento tumoral y metástasis, actuando como genes supresores de tumores o exhibiendo actividad citotóxica directa hacia las células cancerosas. Además, vinculan la respuesta inmune innata 
con la adaptativa y pueden activar inmunidad antitumoral; por lo tanto, la desregulación de las $\beta$-defensinas puede estar asociada con el desarrollo de tumores; ${ }^{63}$ Abiko y colaboradores fueron los primeros en observar alteraciones del patrón de las $\beta$-defensinas humanas en tumores orales, ${ }^{64} \mathrm{y}$ estudios posteriores realizados en 2017 por Sun y su grupo confirmaron el efecto de las $\beta$-defensinas en el desarrollo de tumores orales. ${ }^{65}$ Asimismo, Mizukawa y su equipo mostraron la localización y distribución de las defensinas en el tejido mucoepidermoide de carcinoma oral. ${ }^{51}$

\section{Conclusión}

Se ha demostrado claramente que las defensinas humanas realizan importantes actividades en la defensa de las mucosas y superficies epiteliales del cuerpo a través de la modulación de la respuesta inmunológica; dentro de la cavidad oral, los miembros más comprometidos de esta familia son las $\beta$-defensinas, debido a la alta propiedad antibacteriana contra bacterias en comparación con las $\alpha$-defensinas. Las características descritas en esta revisión bibliográfica hacen a las defensinas extremadamente interesantes; aun cuando son pocos los estudios realizados, la importancia de estas moléculas y su participación en el proceso salud-enfermedad, particularmente en cavidad oral, nos impulsan a continuar descubriendo su relevancia odontológica.

\section{REFERENCIAS}

1. Abbas AK, Lichtman AH, Pillai S. Inmunología celular y molecular. 8a edición. España: Elsevier; 2015. p. 23.

2. Roa NS, Rodríguez A. Inmunidad celular y humoral frente a microrganismos cariogénicos y sus factores de virulencia en caries dental en humanos naturalmente sensibilizados. Univ Odontol. 2013; 32 (69): 61-72.

3. Zlotnik A, Yoshie O. Chemokines: a new classification system and their role in immunity. Immunity. 2000; 12 (2): 121-127.

4. DeFranco AL, Locksley RM, Robertson M. Immunity: The immune response to infectious and inflammatory disease. Yale J Biol Med. 2007; 80 (3): 137-142.

5. Braff MH, Bardan A, Nizet V, Gallo RL. Cutaneous defense mechanisms by antimicrobial peptides. J Invest Dermatol. 2005; 125: 9-13.

6. Schauber J, Gallo RL. Expanding the roles of antimicrobial peptides in skin: alarming and arming keratinocytes. J Invest Dermatol. 2007; 127 (3): 510-512.

7. Koczulla R, Von Degenfeld G, Kupatt C, Krotz E, Zahler S, Gloe $\mathrm{T}$ et al. An angiogenic role for the human peptide antibiotic LL-37/hCAP-18. J Clin Invest. 2003; 111: 1665-1672.
8. Wang G, Li X, Wang Z. APD3: the antimicrobial peptide database as a tool for research and education. Nucleic Acids Res. 2016; 44: 1087-1093.

9. Avila EE. Functions of antimicrobial peptides in vertebrates. Curr Protein Pept Sci. 2017; 18 (11): 1098-1119.

10. Lee SI, Kang SK, Jung HJ, Chun YH, Kwon YD, Kim EC. Muramyl dipeptide activates human beta defensin 2 and proinflammatory mediators through toll-like receptors and NLRP3 inflammasomes in human dental pulp cells. Clin Oral Invest. 2015; 19 (6): 1419-1428.

11. Jenssen H, Hamill P, Hancock REW. Peptide antimicrobial agents. Clin Microbiol Rev. 2006; 19 (3): 491-511.

12. Shai Y. Mode of action of membrane active antimicrobial peptides. Biopolymers. 2002; 66 (4): 236-248.

13. Brogden KA, Ackermann M, Huttner KM. Small, anionic, and charge-neutralizing propeptide fragments of zymogens are antimicrobial. Antimicrob Agents Chemother. 1997; 41 (7): 1615-1617.

14. Téllez GA, Castaño JC. Péptidos antimicrobianos. Infectio. 2010; 14 (1): 55-67.

15. Sang Y, Blecha F. Antimicrobial peptides and bacteriocins: alternatives to traditional antibiotics. Anim Health Res Rev. 2008; 9 (2): 227-235.

16. Dale BA, Tao R, Kimball JR, Jurevic RJ. Oral antimicrobial peptides and biological control of caries. BMC Oral Health. 2006; 6 Suppl 1: S13.

17. Pazgier M, Hoover DM, Yang D, Lu W, Lubkowski J. Human beta-defensins. Cell Mol Life Sci. 2006; 63 (11): 1294-1313.

18. Gallo RL, Hooper LV. Epithelial antimicrobial defense of the skin and intestine. Nat Rev Immunol. 2012; 12 (7): 503-516.

19. Ganz T. Defensins: antimicrobial peptides of innate immunity. Nat Rev Immunol. 2003; 3 (9): 710-720.

20. Castrillón LE, Palma A, Padilla C. Péptidos antimicrobianos: antibióticos naturales de la piel. Dermatología Rev Mex. 2007; 51 (2): 57-67.

21. Dommisch H, Jepsen S. Diverse functions of defensins and other antimicrobial peptides in periodontal tissues. Periodontology 2000. 2015; 69: 96-110.

22. US National Library of Medicine National Institutes of Health. Homo sapiens DEFA/DEFB. PubMed. 2019. [Último acceso el 21 de octubre 2019]. Disponible en: https://www. ncbi.nlm.nih.gov/gene/homosapiensDEFA/DEFB

23. Chen H, Xu Z, Peng L, Fang X, Yin X, Xu N et al. Recent advances in the research and development of human defensins. Peptides. 2006; 27 (4): 931-940.

24. Ganz T, Selsted ME, Szklarek D, Harwig SSL, Deher K, Bainton DF et al. Defensins. Natural peptide antibiotics of human neutrophils. J Clin Invest. 1985; 76 (4): 1427-1435.

25. Dawes C, Pedersen AM, Villa A, Ekström J, Proctor GB, Vissink A et al. The functions of human saliva: A review sponsored by the World Workshop on Oral Medicine VI. Arch Oral Biol. 2015; 60 (6): 863-874.

26. Khurshid Z, Naseem M, Sheikh Z, Najeeb S, Shahab S, Zafar MS. Oral antimicrobial peptides: types and role in the oral cavity. Saudi Pharm J. 2016; 24 (5): 515-524.

27. Sankaran S, Hart R, Dills C. Guardians of the gut: enteric defensins. Frontiers in Microbiology. 2017; 8: 647-654.

28. Lehrer RI, Lu W. a-Defensins in human innate immunity. Immunological Reviews. 2012; 245: 84-112.

29. Falanga A, Nigro E, De Biasi MG, Daniele A, Morelli G, Galdiero S et al. Cyclic peptides as novel therapeutic 
Rev Mex Periodontol 2019; X (3): 77-83

microbicides: engineering of human defensin mimetics. Molecules. 2017; 22: 1217-1232.

30. Nakamura K, Sakuragi N, Takakuwa A, Ayabe T. Paneth cell a-defensins and enteric microbiota in health and disease. Biosci Microbiota Food Health. 2016; 35 (2): 57-67.

31. Mattar EH, Almehdar HA, Yacoub HA, Uversky VN, Redwan EM. Antimicrobial potentials and structural disorder of human and animal defensins. Cytokine Growth Factor Rev. 2016; 28: 95-111.

32. Selsted ME, Tang YQ, Morris WL, McGuire PA, Novotny MJ, Smith W et al. Purification, primary structures, and antibacterial activities of beta-defensins, a new family of antimicrobial peptides from bovine neutrophils. J Biol Chem. 1993; 268 (9): 6641-6648.

33. Diamond DL, Kimball JR, Krisanaprakornkit S, Ganz T, Dale BA. Detection of $\beta$-defensins secreted by human oral epithelial cells. J Immunol Methods. 2001; 256 (1-2): 65-76.

34. Zhang G, Sunkara LT. Avian antimicrobial host defense peptides: From biology to therapeutic applications. Pharmaceuticals (Basel). 2014; 7 (3): 220-246.

35. Klotman ME, Chang TL. Defensins in innate antiviral immunity. Nat Rev Immunol. 2006; 6 (6): 447-456.

36. Scott MG, Hancock RE. Cationic antimicrobial peptides and their multifunctional role in the immune system. Crit Rev Immunol. 2000; 20 (5): 407-431.

37. Harder J, Bartels J, Christophers E, Schroder JM. Isolation and characterization of human beta -defensin-3, a novel human inducible peptide antibiotic. J Biol Chem. 2001; 276 (8): 5707-5713.

38. Coretti L, Natale A, Cuomo M, Florio E, Keller S, Lembo $\mathrm{F}$ et al. The interplay between defensins and microbiota in Crohn's Disease. Mediat Inflamm. 2017; 2017: 8392523.

39. Zhu BD, Feng Y, Huang N, Wu Q, Wang BY. Mycobacterium bovis bacille Calmette-Guérin (BCG) enhances human beta-defensin-1 gene transcription in human pulmonary gland epithelial cells. Acta Pharmacol Sin. 2003; 24 (9): 907-912.

40. Yang D, Liu ZH, Tewary P, Chen Q, de la Rosa G, Oppenheim JJ. Defensin participation in innate and adaptive immunity. Current Pharmaceutical Design. 2007; 13: 3131-3139.

41. Cagliani R, Fumagalli M, Riva S, Pozzoli U, Comi GP, Menozzi $\mathrm{G}$ et al. The signature of long-standing balancing selection at the human defensin $\beta-1$ promoter. Genome Biology. 2008; 9 (9): 143-154.

42. Sayama K, Komatsuzawa H, Yamasaki K, Shirakata Y, Hanakawa Y, Ouhara K et al. New mechanisms of skin innate immunity: ASK1-mediated keratinocyte differentiation regulates the expression of b-defensins, LL37, and TLR2. Eur J Immunol. 2005; 35 (6): 1886-1895.

43. Fattorini L, Gennaro R, Zanetti M, Tan D, Brunori L, Giannoni $\mathrm{F}$ et al. In vitro activity of protegrin- 1 and beta-defensin-1, alone and in combination with isoniazid, against Mycobacterium tuberculosis. Peptides. 2004; 25: 1075-1077.

44. Sun L, Finnegan CM, Kish-Catalone T, Blumenthal R, Garzino-Demo P, La Terra Maggiore GM et al. Human beta-defensins suppress human immunodeficiency virus infection: potential role in mucosal protection. J Virol. 2005; 79 (22): 14318-14329

45. Prado E. Defensinas humanas: ¿profilaxis y terapia contra el VIH? Gac Méd Méx. 2006; 142 (5): 431-433.
46. Bullard RS, Gibson W, Bose SK, Belgrave JK, Eaddy AC, Wright CJ et al. Functional analysis of the host defense peptide human beta defensin-1: new insight into its potential role in cancer. Mol Immunol. 2008; 45: 839-848.

47. Diamond G, Beckloff N, Weinberg A, Kisich KO. The roles of antimicrobial peptides in innate host defense. Curr Pharm Des. 2009; 15 (21): 2377-2392.

48. Sorsa T, Gursoy UK, Nwhator S, Hernandez M, Tervahartiala $\mathrm{T}$, Leppilahti J et al. Analysis of matrix metalloproteinases in gingival crevicular fluid (GCF), mouthrinse and saliva for monitoring periodontal diseases. Periodontology 2000. 2016; 70: $142-163$.

49. Zhao C, Wang I, Lehrer RI. Widespread expression of B-defensin hBD-1 in human secretory glands and epithelial cells. FEBS Letters. 1996; 396: 319-322.

50. Diamond G, Beckloff N, Ryan LK. Host defense peptides in the oral cavity and the lung: similarities and differences. J Dent Res. 2008; 87 (10): 915-927.

51. Mizukawa N, Sugiyama K, Ueno T, Mishima K, Takagi S, Sugahara T. Defensin-1, an antimicrobial peptide present in the saliva of patients with oral diseases. Oral Dis. 1999; 5: 139-142.

52. Sawaki K, Mizukawa N, Yamaai T, Yoshimoto T, Nakano M, Sugahara T. High concentration of betadefensin- 2 in oral squamous cell carcinoma. Anticancer Res. 2002; 22 (4): 2103-2107.

53. Bonass WA, High AS, Owen PJ, Devine DA. Expression of $\beta$-defensin genes by human salivary glands. Oral Microbiol Immunol. 1999; 14 (6): 371-374.

54. Bals R, Wang X, Wu Z, Freeman T, Bafna V, Zasloff M et al. Human $\beta$-defensin 2 is a salt-sensitive peptide antibiotic expressed in human lung. J Clin Invest. 1998; 102 (5): 874-880.

55. McKay MS, Olson E, Hesla MA, Panyutich A, Ganz T, Perkins $S$ et al. Immunomagnetic recovery of human neutrophil defensins from the human gingival crevice. Oral Microbiol Immunol. 1999; 14 (3): 190-193.

56. Navarra CO, Robino A, Pirastu N, Bevilacqua L, Gasparini P, Di Lenarda $R$ et al. Caries and innate immunity: DEFB1 gene polymorphisms and caries susceptibility in genetic isolates from north-eastern ital. Caries Research. 2016; 50: 589-594.

57. Goeke E, Kist S, Schubert S, Hickel R, Huth K, Killmuss M. Sensitivity of caries pathogens to antimicrobial peptides related to caries risk. Clin Oral Investig. 2018; 22 (7): 2519-2525.

58. Lee JK, Chang SW, Perinpanayagam H, Lim SM, Park YJ, $\mathrm{HanSH}$ et al. Antibacterial efficacy of a human $\beta$-defensin-3 peptide on multispecies biofilms. J Endod. 2013; 39 (12): 1625-1629.

59. Farges JC, Alliot-Licht B, Renard E, Ducret M, Gaudin A, Smith AJ et al. Dental pulp defence and repair mechanisms in dental caries. Mediators Inflamm. 2015; 2015: 230251

60. Hahn CL, Best AM, Tew JG. Cytokine induction by Streptococcus mutans and pulpal pathogenesis. Infect Immun. 2000; 68 (12): 6785-6789.

61. Hosokawa Y, Hirao K, Yumoto H, Washio A, Nakanishi T, Takegawa D et al. Functional roles of NOD1 in odontoblasts on dental pulp innate immunity. Biomed Res Int. 2016; 2016: 9325436.

62. Cooper PR, Holder MJ, Smith AJ. Inflammation and regeneration in the dentin-pulp complex: a double-edged sword. J Endod. 2014; 40: 46-51. 
Rev Mex Periodontol 2019; X (3): 77-83

63. Nishimura E, Eto A, Kato M, Hashizume S, Imai S, Nisizawa $\mathrm{T}$ et al. Oral streptococci exhibit diverse susceptibility to human $\beta$-dedensin-2: antimicrobial effects of hBD-2 on oral streptococci. Curr Microbiol. 2004; 48: 85-87.

64. Abiko Y, Suraweera A, Nishimura M, Taishin TA, Mizoguchi I, Kaku T. Differential expression of human beta-defensin 2 in keratinized and non-keratinized oral epithelial lesions: immunohistochemistry and in situ hybridization. Virchows Arch. 2001; 438: 248-253.

65. Sun CQ, Arnold RS, Hsieh CL, Dorin JR, Lian F, Li Z et al. Discovery and mechanisms of host defense to oncogenesis: targeting the B-defensin-1 peptide as a natural tumor inhibitor. Cancer Biol Ther. 2019; 20 (6): 774-786.

Correspondencia:

Rocío Patricia Mariaud Schmidt

Instituto de Investigación en Odontología, Centro Universitario de Ciencias de la Salud, Universidad de Guadalajara.

José Ma. Echauri s/n, Esq. Salvador de Quevedo y Zubieta, Edificio C, primer piso. E-mail: rpmariaud@gmail.com 